\title{
The Control of Powerful Neutral Beams
}

\author{
Edward Theil \\ Van Jacobson \\ Real Time Systems Department \\ Lawrence Berkeley Laboratory \\ University of California \\ Berkeley, CA 94720
}

May 2, 1986

\begin{abstract}
DISCLAIMER
This report was prepared as an account of work sponsored by an agency of the Uniled States Government. Neither the United States Government nor any agency thereof, nor any of their employecs, makes any warranty, express or implied, or assumes any legal liability or responsibility for the accuracy, completeness, or usefulness of any information, apparatus, product, or process disclosed, or represents that its use would not infringe privately owned rights. Reference herein to any specific commercial product, process, or service by trade name, trademark, manufacturer, or otherwise does not necessarily constitute or imply its endorsement, recommendation, or favoring by the United States Government of any agency thereor. The views and opinions of authors expressed herein do not necessarily state or reflect those of the United States Government or any agency thereol.
\end{abstract}

This work was supporled by the Director, Office of Energy Research, Office of High Energy and Nuclear Plysics. Nuclear Physics Division, U.S. Department of Energy, under Contract Number DE-AC03-76SF00098. 


\title{
The Control of Powerfui Neutral Beams
}

\author{
Van Jacobson \\ Edward Theil \\ Real Time Systems Group \\ Lewrence Berkeley Labontory \\ University of California \\ Berkeley, California 94720
}

May 2,1986

\begin{abstract}
While significant progress has been made in the development of neutral beams for the heating and sustaining of plasmas in large fusion experiments, the control of such devices has largely been a matter of hardware interlocks and operator experience. The need for computer-assisted control becomes more evident, however, with the initiation of multi-beamline experiments.

This paper describes a software system that incorporates simple mathematical models coupled to Kalman filters for control of the high power (6 to $8 \mathrm{MW}$ ) beams currently under development at Lawrence Berkeley Laboratory's Neutral Beam Engineering Test Facility. Among the principal features of the system are: reduction of a large number of operator variables to just a few (usually one or two); the ability to describe most of the major neutral beams in use and under development; a foundation resting on statistical data analysis and control system principles rather than rules-ofthumb.
\end{abstract}

\section{Introduction}

The development of high power ion sources for neutral beam heating of plasmas presents a number of interesting challenges in applied physics and engineering. The particular problem we wish to address here is the control of the source. Relatively little work has been done in this area (exceptions noted in Section 8), since the emphasis has naturally been on the research and development of the ion sources themselves, and the construction of the beamlines that form the delivery system (see [1] and its bibliography). Nevertheless, the utility of attomatic control becomes clear once one moves from the development and testing of a single source to the role that the bearns must play in fusion devices.

Complex as the operation of a single high-powered source is, that complexity will be magnified by the twelve beams at TFTR or the dozens of beams at MFTF-B, to cite just two prominent examples. As a rule, neutral beams need frequent "conditioning", a process whereby they are brought from low to high voltage over a long period of shots. Thus, a large fusion experiment will have both injeccion and conditioning shots occurring in parallel. The resulting drain on the experiment's human and system resources can obviousiy become critical. 
We have developed a computerized system that provides a variety of aids for operating the source while, at the same time, supplying diagnostic information ranging from simple graphical displays of temperatures and waveforms to analyuic models of beam profiles and archived data analyses. In this paper we have tried to abstract just those characteristics of the system that are important for a high level of control while ignoring the hardware and implementation details that seem less central to an understanding of the system at a conceptual level. Other examples of integrated diagnostics and control in the system are given in [2] and [3].

\section{Goals fo: the system and outine of an approach}

Stated briefly, the uitimate goal of the control system is to reduce the many parameters presented to the neutral beam operator to as few as practical. Since there are separate power supplies (and therefore a current and a voltage to be specified) for the filaments, the arc, the accelerator grids, the gradient (second) grids and the suppressor (third) grids, there are ten values for each source's electrical requirements. We shall see that six of these are critical. A more immediate goal, then, is to search for dependencies among these variables in order to reduce the choices. The presence of secondary variables (e.g., gas flow) can affect these dependencies, which will be only partially understood at best.

As is often the case in such situations, one first atempts to build simple models of ion source behavior. For example, it seems evident that arc current and voltage are related in some consistent fashion. A simple relationship based on empirical evidence suggests itself:

$$
V_{\mathrm{xrc}}=\rho I_{\mathrm{arc}}+\beta
$$

The units of $\rho$ suggest the name "arc impedance." Unfortunately, this model is too simple to be very useful by itself, since the unknown parameters $\rho$ and $\beta$ depend on recent perturbations in other source elements (such as a change in filament temperature) that aff -at the plasma generator. Consequently, a second element of the modeling process, estimation of the parameters as they change in time, is necessary. The description of source behavior, then, is in two parts: source models that state how we think things work in general, and dynamic estimators that tell us how things are working in particular.

The remainder of this paper proceeds as follows. First, we describe the set of source models that statically represent the relationships between various source components. Then the specific estimation tool -Kalman filters -- used to supply the model parameters is discussed. These filters have the function of incorporating past history into the source models. With the models and estimators in place, the simple algorithms to produce setpoints are easily and briefly described. A concluding section describes results based on actual test stand data from two different facilities, as well as the applicability of this scheme to a variety of neutral beam designs.

\section{The Source Models}

Details of source design and operational characteristics have been described elsewhere [4]. Very briefly, the major (but by no means only) components of a neutral beam are the filamerits, the arc subsystem and the accelerator. The first two are the principal elements of the jon source, while the third forms the beam and energizes the particles. 


\section{Filament Model}

Taken in isolation, the filament may be modeled as the simplest kind of electrical circuit,

$$
V_{\beta^{1}}=a_{j !}^{\prime}+b
$$

where it is natural wo think of $a$ as the "filament impedance." (Here, and in what follows, we adopt the convention of naming signals by using $I$ and $V$ for current and voltage, followed by a subscript for the particular subsystem). Typical values for $I_{f l}$ and $V_{f i l}$ are about 4000 amps and 10 volts. ("Typical" values are representative of current designs at $\mathrm{LBL}$ ).

(3.1) is not an adequate model, however, when the arc is discharged. The purpose of the arc is to provide sufficient voltage, in conjunction with the thermoelectrons, to ionize the source gas. But the existence of a large arc current supplies addjtional electrons at the negative leg of the filaments, causing that end to heat up. As a consequence, the measured filament current increases as the square root of the temperature rise while the filament voltage drops. Thus the same filament power supply settings with and without arc can produce distinctly different (and misleading) data.

To compensate for the arc's effect on the filament, (3.1) is extended by a linear term in arc current:

$$
V_{f i}=a J_{f l}+b+c I_{2 \pi}
$$

\section{Arc Model}

The interaction between the arc and the other components is a complex one which we summanize only briefly. The arc may operate in either of two modes, "space-charge limited" and "emission-limiud". In the former, the rate at which ions are produced from the source gas by the arc discharge limits the rate at which electrons are attracted from the filaments; changes in the arc potential therefore produce roughly proportional changes in the arc current. Thus, the simple equation $V_{\text {wr }}=d I_{\text {arc }}+e$ can serve as an adequate model.

In emission-limited mode, the filaments are producing as many electrons as possible for a given flament current and the limiting factors are thermal. In this mode, the flament has a strong effect on the behavior of the arc discharge, since the degree of ionization and thus the arc current depends on the filament's supply of electrons. It follows that an increase in arc voltage will tend to produce little additional arc current, since no additional electrons are available. However, an increase in filament current, causing a hotter cathode, will increase the electron supply. Thus, one needs less arc, potential to achieve the same arc current.

Typically, "field-free" sources, such as those in use at Doublet III and planned initially for TFTR, operate in the space-charge limited mode, while "magnetic bucket" sources, which produce more of the full energy and less of the heavier ions, operate in emission-limited mode. Generalized models for both types of plasma generators have been deyeloped at LBL. However, for simplicity in exposition and because we have not yet had the opportunity to test the system on a bucket source, this discussion is confined to the field-free type. In that case, the model is simply

$$
V_{\mathrm{art}}=d I_{\mathrm{arc}}+e
$$


Operating data shows that the arc's behavior is not linear over the entire range of interest but because the parameters are treated as changing in time, the locally linear representation (3.3) is adequate, as explained more fully in the next section.

\section{Accel Models}

There are two accel models. The first describes the relation between beam current, $I_{\text {accal }}$, and the arc. We have found empirically that the relationship between arc power and $I_{\text {accel }}$ tends to be

$$
P_{\text {are }}=f l_{\text {accel }}+g
$$

to a good approximation.

The second model derives from the well known Child-Langmuir law that governs the relationship between the accel voltage and the beam current:

$$
I_{\text {accel }}=p V_{\text {accel }}^{15}
$$

$p$ is the perveance for the shot. Since the ion beam contains three species of hydrogen or deuterium, $p$ will in general depend on the relative percentage of each specie. Although there is an optimum perveance for any $V_{\text {accel }}$, we shall treat $p$ as a variable that allows us to control the beam current for a given voltage.

Taken together, (3.2)-(3.5) describe the critical relationships needed to predict and control source behavior. For easy reference, we reproduce all the equations here.

$$
\begin{gathered}
V_{A I}=a I_{a}+b+c I_{\text {wrc }} \\
V_{\text {urc }}=d I_{a x c}+e \\
P_{\text {ax }}=f I_{\text {accel }}+g \\
I_{\text {accel }}=p V_{\text {accel }} 1.5
\end{gathered}
$$

We refer to all the parameters $(a, b, \ldots, g, p)$ as the source parameters.

In addition to these, there are control parameters for the second and third grids. They are much simpler, because they change infrequently. For example, both $V_{B g}$, the second grid voltage, and $V_{\text {app }}$, the third grid voltage, are kept at specified percentages of $V_{\text {accel }}$. Consequently, these parameters do not present any particular difficulties and we shall not refer to them again.

We note in passing that the models for both the field-free and magnetic bucket sources have the same overall structure, although the latter have more equations and each is multi-dimensional

\section{Estimation of Source Parameters by Kalman Filtering: Source Behavior as a Stochastic Process}

Earlier, we pointed out that the absence of specific variables such as gas pressure or type does not mean that they are unaccounted for in the models. Instead, we view the source parameters as changing in time and subject to a number of unspecified and essentially random influences (among which is gas flow). Informally then, the time evolutions of the model parameters are treated as stochastic processes. Even though many of the disturbances are not truly rarjom, we can model them as part of the intrinsic noise of 
the system as a whole.

At the same time, the discrete, pulsed nature of the beam operation suggests that a form of sequential data filtering using difference equations would be a natural technique. The specific tool chosen for the estimation process is Kalman filtering. ([5], [6], [7] are three useful references listed in order of increasing mathematical sophistication).

In addition to these heuristic arguments, a substantial body of theory indicates that Kalman filters have the desirable properties of optimality (in the sense of minimum enor variance, for example) and robustness (in the sense of good suboptimal performance) needed in the present application. Finally, we found, by direct experiment, that Kalman filtering provided estimates that were more realistic physically than did other less sophisticated methods, such as the related technique of sequential "finite memory" least squares.

The discrete Kalman filter is a digital filter of the predictor/correction form, where the prediction of a parameter before data taking is corrected after data taking by a term depending on the residual; i.e., the difference between the prediction and measured value. Unlike a conventional digital filter, however, the correction term is weighted by a factor that incorporates estimates of both the system (or "plant") noise and measurement noise, and these weights can and $d r$ change in time. In the present case, both the filter formula and its implementation are particularly simple.

To be specific, consider just the filament model. This time we have added the subscript and argument $n$ to refer to the values of the measurements and parameters just after the nth beam shot.

Let $X(n)$ represent the vector of model parameters:

$$
X(n)=\left[\begin{array}{l}
a_{n} \\
b_{n} \\
c_{n}
\end{array}\right]
$$

and $M(n)$ the row vector of measurements on the ath shot:

$$
M(n)=\left[I_{f i}(n), 1, I_{n r c}(n)\right]
$$

Our estimate of the vector $X(n+1)$, based just on krowledge of the vector $X(n)$, is simply

$$
X(n+1)=X(n)+w(n)
$$

where $w(n)$ is a vector of (system) white noise with given covariances. Observe that if $w(n)=0$, then we are assuming that the parameters do not change in time. ${ }^{1} X(n)$ represents the state of the filament system, which is driven by white noise; i.e., undergoes a random walk.

(4.2) says that the expected values of the filament parameters will be the same on the next shot as on the last shot ( $\bar{w}=0$ by assumption), but the values are subject to a variety of influences whose magnitudes are contained in the covariance matrix of $w$, a matrix we shall call $Q(n)$. We cannot know these values precisely, but we car make informed estimates and modify them on the basis of desired control criteria, or make them self-modifying (adaptive filtering) af ter each shot.

'In classical least squares, $w(n)=0$. 
We must also express the relationship between the measurements and the estimated parameters. Based on (3.2) and (4.2), we dejine

$$
V_{f}(n)=\langle M(n), X(n)>+v(n)
$$

where $v(n)$ is white noise with variance $R(n)$ in the measurement process. $(<,>$ denotes the usual scalar product of vectors). In this simple case, $v$ is of course a scalar. Note that $V_{f}$ is the model estimate of $V_{f i}$. Again, we must estimate $R$. (The process of estimating and modifying $R$ and $Q$ is called "tuning the filter") ${ }^{2}$

Now suppose we have estimates of $a_{n}, b_{n}$ and $c_{n}$ prior to the nth shot. The shot is taken and the estimates need to be updated. In the predictor-correction form, the Kalman filter estimates will be

$$
X(n+1)=X(n)+K(n)\left[V_{f l l}(n)-V_{f}(n)\right]
$$

Note that the new estimate is the old estimate, modified by the error from the last shot weighted by the vector $K(n)$. The latter is referred to as the "filter gain".

$K(n)$ is a function of both the system and the measurement noise. Since space does not permit a full derivation of the recursion equations used to update the filter, we refer interested readers to [5] and present only an intuitive interpretation of $K$ in a general but one-dimensional case.

Recalling that $Q$ is the variance of the system noise and $R$ the variance of the measurement noise, the expression for a one-dimensional $K$ will reduce to

$$
K(n)=\frac{Q(n)}{Q(n)+R(n)} .
$$

Two extreme cases are possible. If $Q=0$, then $K=0$ and the parameters do not change in time. Then the data model in (4.4) is ignored. If $R=0$, then $K=1$. This corresponds to complete confidence in the data model and none in the system model, with $X(n)$ adjusted by the full residual on each shot. Obviously, "tuning" the filter requires choosing values for $R$ and $Q$ in a manner that will optimally weight the correction in (4.4). Figure 1 is a block diagram of the filament model with filter. Each model (3.2)-(3.4) has a similar filter structure.

Returning to the general case, it should be evident that, depending on the values of the covariances in the matrices $Q$ and $R$, the filter will respond more or less rapidly to incoming data. As with any filter, the problem of distinguishing between noisy measurements and "real" values is always present In the original Kalman formulation, there is no feedback from the state equations (4.2) to the covariance updates. The fitter has no way of knowing, for example, if the flow of source gas is changed significanily; it can only respond to changes in measured values at a rate controlled by $Q$ and $R$.

It is possible, however, to add features that make the filters adapt more rapidly to changing conditions. Perhaps the most obvious way is to examine the residuals -- the difference between predicted and measured values - after each shot. Under optimal conditions, the residuals are normally distributed with mean 0 and a known variance which changes in time. Therefore, various nules can be established that help to distinguish noise from real data, much as in sequential decision making. For example, a typical rule might be: if

${ }^{2}$ One way of vicwing the Kaiman filter is that it is the minimum variance sequential least-squares estimator of $X(n)$ subject to the constrints (4.2) and (4.3) 
two consecutive residuals have the same sign and exceed 2 standard deviations in magnitude, increase the filter gain by a predetermined amount and recalculate (4.4) until the latest residual drops to within 1 standard deviation. 3

The neutral injector itself, however, generally needs time (several shots) to "adjust" to changing operating conditions. Therefore, the filters have been able to track its behavior satisfactorily without this additional feature.

\section{Controlling the Source}

As stated earlier, a principal goal has been to reduce the number of degrees of freedom in operating the source from many to few. For the great majority of beam shots, the key variable is accel voltage. ${ }^{4}$ Once the models are in place and the filter estimates are available, the implementation of control is deterministic and straightforward. Given that a certain accel voltage $V_{\text {occel }}$ is desired,

$$
I_{\text {accel }}=p V_{\text {accel }}{ }^{1.5}
$$

and then

$$
P_{\text {nct }}=f I_{\text {acest }}+g
$$

To determine $I_{\text {arc }}$, recall

$$
V_{a x}=d I_{a r c}+e .
$$

To preserve arc power and thus produce the correct beam current, (5.2) with $P=I V$ yields

$$
P_{\text {arc }}=d I_{\text {arc }}^{2}+e l_{\text {arc }}
$$

from which one easily determines first $I_{a x}$ and then $V_{a r}$.

For LBL field-free sources, the filament settings normally do not change. This reflects the space-charge limited mode, where the supply of electrons is normally sufficient to fully ionize the plasma. Although the model (3.1) still holds, the values for $I_{f i}$ or $V_{f i}$ are ordinarily held fixed. Thus, if $I_{f l}$ is to remain consrant, (3.1) can be used to determine $V_{f i l}$. Of course, this will not be true in emission-limited mode, where the arc and filament models are coupled more strongly.

It was implicitly assumed above that the perveance $p$ was given. If not, it 100 will be an input variable. (The optimum perveance -- that value of $p$ resulting in minimum beam divergence -- can ide determined by employing another part of the diagnostic system [9]). The other principal input specification for the beam is the duration of the shot. The timing subsystem, which is independent of the source power requirements, controls and automatically adjusts the duration of various signals depending on the beam duration. Neutral beam timing control is described in [10].

To use the system, the beam operator turns a knob whose values are interpreted by the control computer in units of $V_{\text {accel }}$. He then presses a "set power supplies" button, which serves as a signal to the

'For a further discussion of this point, see [8]

"Actually, it is usually convenient to control either voltage or accel current. We omit the delails in the latter case, since it is a simple variant on the scheme described here. 
computer to perform the algorithm described above and to send the resul ing $(l, V)$ values to a satellite system whose sole job it is to translate those values into the units of the local power supplies and then arm the supplies (bring them to their setpoints). This translation, from engineering units to local units is independent of any of the control features discussed above

\section{Results and Applicability}

The source model approach to beam control has been used to operate the $120 \mathrm{KV}$ field-free source at LBL. In all cases, the models performed satisfactorily. By that we mean the deviation from requested source values for arc and accel averaged 1 to $3 \%$ with maximum errors of about 5\%. An example of their use was during an automatic conditioning tun, when the beam was brought from 90 to $110 \mathrm{KV}$ over a period of approximately 60 shots, with no operator intervention, except to tune the source by varying the perveance at the $100 \mathrm{KV}$ level. Figure 2 is the computer display used to monitor the models and show the results of the last shot. The graphs in that figure show equations (3.3)-(3.5) with recent shots plotted. The table displays the latest set of requested values with the resulting entor relative to measured values after the shot. Typically, the operator will adjust only $v_{\text {accel }}$ or perveance.

To illustrate filter performance in greater detail, we use a recent study [11] based on actual test stand data from Lawrence Livermore Laboratory's MFTF Neutral Beam Test Stand. The analysis was done offline, so the values predicted by the filters were not used to control the source. Nevertheless, the distribution of errors shown in Figures 3 and 4 are a good indication of the filter performance had the source models been on-line at that time. (By contrash, most such studies rely on some form of computer generated randomness to simulate the noise.)

T7. measured power supply averages were taken during a run in which a short pulse ( 0.5 second) neutral beam injector was conditioned from 30 to $80 \mathrm{Kv}$. For the purposes of the study, $I_{\text {occal }}$ was taken as the primary control variable. That is, (3.5) was treated as given by looking ahead at the data, instead of using a knob for $I_{\text {accel }}$.

The control system was required to predict the values of $P_{a \pi}, I_{\mathrm{arc}}$ and $V_{\mathrm{ar}}$ for the next shot. These predictions were then compared with their measured counterparts for that shot. The results for just $V_{\text {arc }}$ are shown in the histogram and graph (all the errors are relative, i.e., $\frac{\left(V_{p r e d}-V_{\text {meas }}\right)}{V_{\text {mear }}}$, expressed as percentages). The graphs for $I_{a \pi}$ and $P_{a \tau}$ are entirely similar. Additional details are available in [11].

Table 1 suinmarizes all the results in terms of the average error, standard deviation, and the largest ne 6 acive and positive errors (for 700 shots). The average error is very small, showing that the filters produce unbiased estimates of the signals. The most important statistic is the standard deviation, which confirms the listogram of Figure 3. About $95 \%$ or more of the errors will be less than 4\%. Again, all values are percentages of the measured quantities. 


\begin{tabular}{|c|c|c|c|c|}
\hline \multicolumn{5}{|c|}{ Summary of Prediction Errors } \\
\hline Signal & Avg. Error & Std. Dey. & $\operatorname{Max}$ & Error \\
\hline$P$ anc & -0.025 & 1.49 & -8.0 & 5.9 \\
\hline$V$ arc & -0.012 & 1.10 & -6.5 & 5.9 \\
\hline I arc & -0.016 & 1.24 & -7.0 & 7.6 \\
\hline
\end{tabular}

Table 1.

It appears that the shon pulse injectors at MFTF-B are good candidates for the type of control system described here and, in fact, work is currently under way to implement this scheme at MFTF-B.

Finally, some remarks about generality are in order. The extension of the source models to describe both field-free and bucket sources should be sufficient to estimate parameters for most, if not all of the major neutral beams under development today. For example,

(1) LBL field-free for TFTR: the models have proved effective, as just described.

(2) Doublet III: the beams are based on the LBL design.

(3) LBL Iong pulse "bucket": we have tested the generalized models on archived data with satisfactory results, although full, closed loop control is not part of the NBETF program. The Common Long Pulse Source (CLPS) is similar in its operation to this one.

(4) JET neutral becms: these have bucket type plasma generators, similar to (3), but with $V_{f l}$ or $I_{f l}$ fixed. This constraint shou!d tend to simplify control, because the arc and flament models will have fewer degrees of freedom.

(5) MFTF-B: Results for the 0.5 second sources are described above. Work is under way at LLL to implement the Kalman filter approach for actual control. The long puIse sources will be based or the CLPS tesign, and it is anticipated that they will behave as in (3).

\section{Discussion}

We have presented a scheme for control of neutral beam injectors that uses a feedback mechanism ir the form of Kalman filters. The results of the previous section suggest to us that it can be employed effectively in a number of fusion experiments.

A natural alternative to the approach described here is to store tables of previously measured power supply settings in a computer and use table lookup to obtain an n-tuple of setpoints under currently prevailing conditions. While this is indeed straightorward, it implicidy assumes that conditions at one point in time are reproducible at a later time for the same accel voltage. Unfortunately, this is not the case in an environment where hardware configurations and numerous operating parameters frequently change. We prefer to let the filters "learn" the current operating regime. 
To put it another way, the use of stored tables is essentially open lowp control, while the nethod described in this paper is closed loop (feedback). It is well knoun that the latter is iaherently niore stable.

\section{Acknowledgements}

Other work in this field includes $[3,12,13]$. [12] is similar in spirit and technique to this report and it is a pleasure to acknowledge our debt to that earlier work. [13] is an interesting discussion of control systems in general, while [3] contains some of the principles that have guided our own work.

Finally, we thank Klaus Berkner and Alin Lietzke for many enlightening conversations on all aspects of neutral beams. Our mistakes are our swn, but our knowledge is largely theirs. 
9. References

1. Development of $120 \mathrm{Kev}$ Neutral Beam Injectors, K. H. Berkner et a., 8th Symposium on Engineering Problems in Fusion Research, San Francisco. 1979

2. Progress in Computer-Assisted Diagnosis, Control and Conditioning of Neutral Beams E. Theil et al., 11th Symposium of Fusion Technology, Oxford, 1980 (LBL Repor LBL-11839)

3. A Very High Level Control System for Neutral Beams, V. Elischer, V. Jacobson, E. Theil, 9th Symposium on Engineering Problems of Fusion Research, Chicago, 1981 (LBL report LBL-13491)

4. Rectangularly Shaped Large Area Plasma Source, K. W. Ehlers, 6th Symposium on Engineering Problems of Fusion Research

5. Digital and Kalman Filtering, S. M. Bozic, Halstead Press, 1979

6. Applied Optimal Estimation, A. Gelb, Ed., MITT Press, 1974

7. Stochastic Models, Estimation and Control, P. S. Maybeck, Academic Press, 1980

8. A Robust Sequential Projection Algorithm for Traffic Load Forecasting, Bell System Technical Jouma, V. 61, 1, January, 1982

9. A User's View of the Source Tuning Programs at NBETF, LBL Repon LBID-577, 1982

10. Neutral Beam Timing, LBL Report LBD-579, 1982

11. Simulation of Automatic Control of MFTF-B Neutral Beans, E. Theil, LBL Repor LBL-19252, 1985

12. Computer Assisted Operation of Neutral Beam Injectors, R. Metzinger and D. Keene, Sixth Symposium on Engineering Problems of Fusion Research, San Diego, 1975

13. Towards a Generalized Computer Control System: An Analysis, G.Sprechert, C. Grant and D. Gruenther, Lawrence Livermore Laboratory, UCRL 86339, 1981 and 9th Symposium of Engineering Prob'ems of Fusion Research, Chicago, 1981 


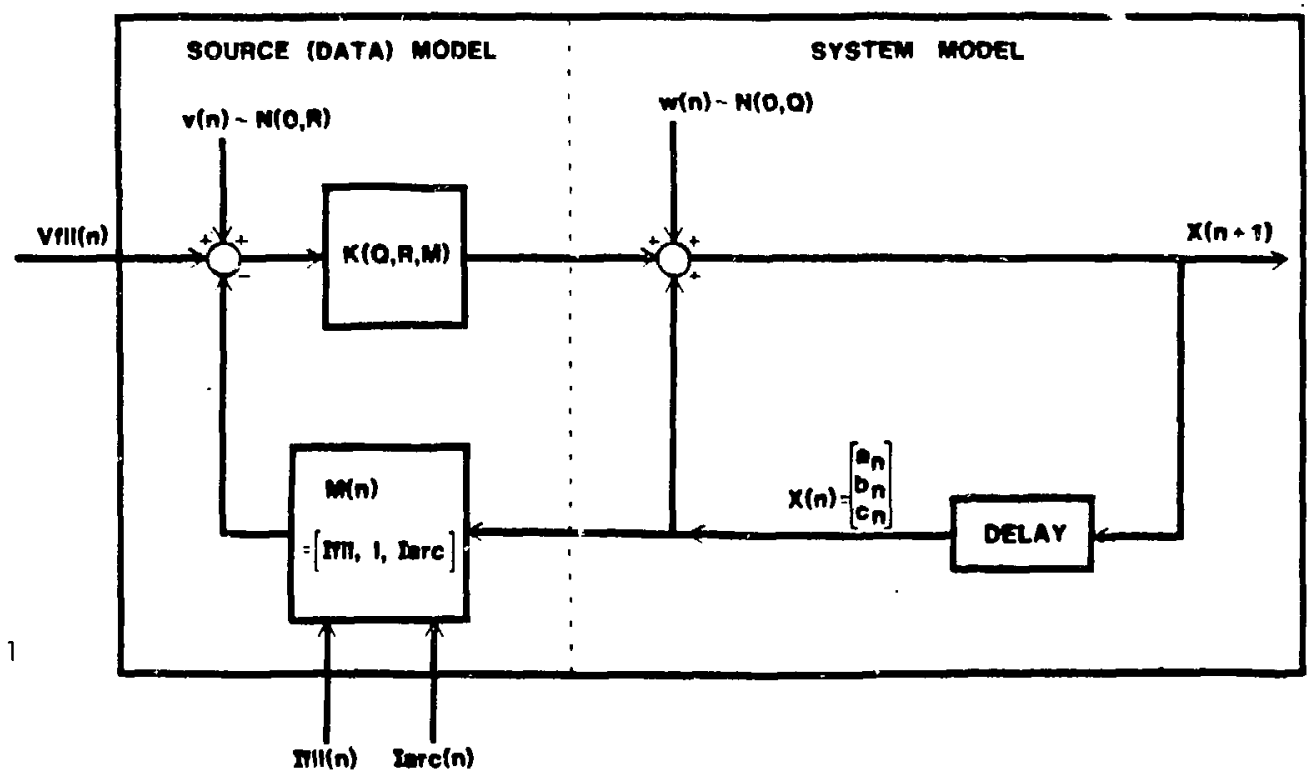

BLOCK DIAGRAM OF THE FRAMENT ESTMATOA 
PQUER SUPPLY CONTROLS

Y-ape us. I-arc

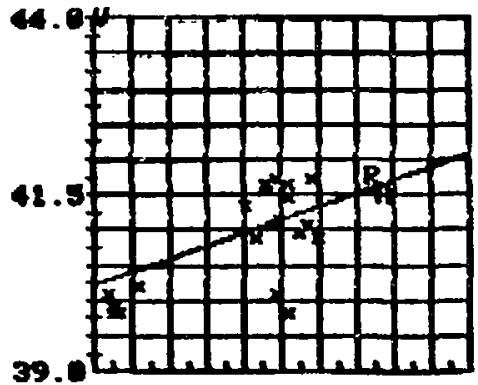

mi. 4 ex
$1 . \operatorname{sen}$
- Rי - REC:

in' -O MSKE d-accel us firc Poner

55.0

6.0

Ape Ieped 9.2700

firc etf "s . S4carku

Perveanes 1.47up
45.

KUS6.0K
$61.0 x$

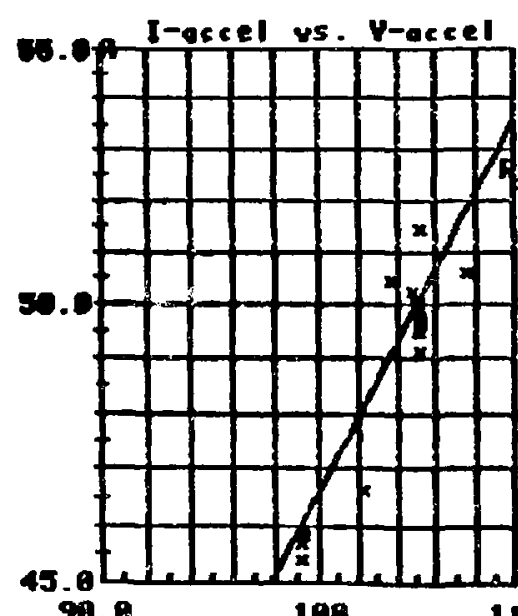

\begin{tabular}{|c|c|c|c|c|c|}
\hline & $\begin{array}{l}\text { Current } \\
\text { Setpts }\end{array}$ & $\begin{array}{l}\text { Deviet } \\
\text { R-n }\end{array}$ & $\operatorname{lons}^{2}$ & $\begin{array}{c}\text { Correc } \\
\text { Abs }\end{array}$ & $\begin{array}{l}5 \\
x\end{array}$ \\
\hline Perv & $1.43 u P$ & $18.46 P$ & 1. & & \\
\hline Yacl & $112 K 4$ & $-2.29 k \%$ & -2 & $3.63 \times 4$ & \\
\hline Iex & $53.6 \mathrm{R}$ & $-967=9$ & -2. & 1E. Tan & \\
\hline Pape & 69.7N.11 & $-3.99 k 1$ & -6 . & $4.12 \times M$ & $B$ \\
\hline $10: 1$ & on & $4.29 \times 9$ & $\neq 88$. & & \\
\hline 142 & $a x$ & 15.07 & $\$ 00$. & & \\
\hline
\end{tabular}



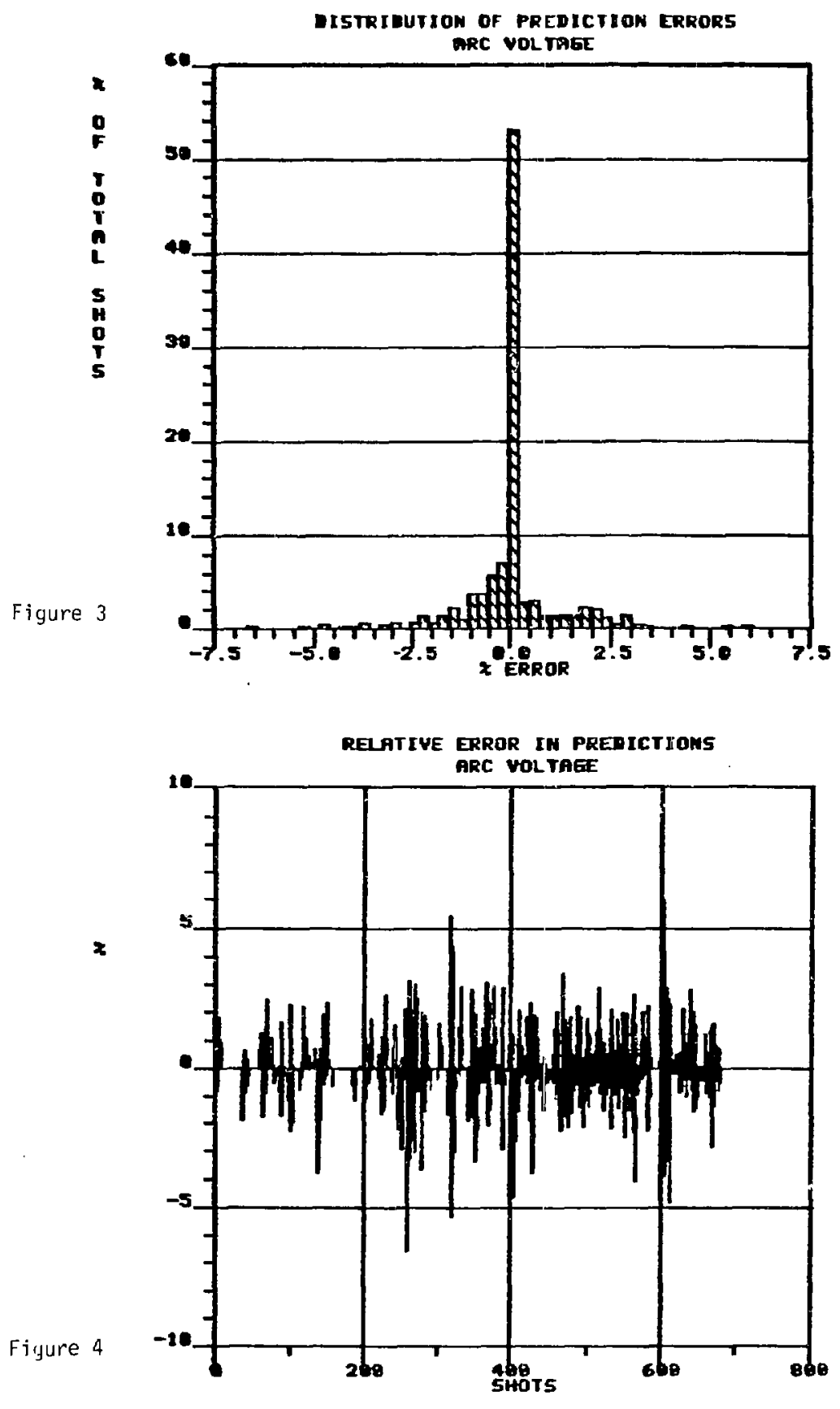
This repon was done with suppon from the Department of Energy. Any conclusions or opinions expressed in this report represent solely those of the author(s) and not necessarily those of The Regents of the University of California, the Lawrence Berkeley Laboratory or the Department of Energy.

Reference lo a company or product name does not imply approval or recommendation of the producl by the University of Califorma or the U.S. Department of Energy to the exclus son of others that may be suitable. 